\begin{tabular}{|c|c|c|c|}
\hline \multicolumn{2}{|c|}{$\begin{array}{l}\text { Datum in m.Z. } \\
\text { Greenwich }\end{array}$} & $x$ & $y$ \\
\hline \multicolumn{2}{|c|}{1921} & - & - \\
\hline \multirow[t]{2}{*}{ Juli } & 7.208 & $675.5^{\circ}$ & $327: 8.9$ \\
\hline & 8.205 & .65 & .63 \\
\hline & 9.203 & .79 & .36 \\
\hline & 10.200 & .92 & .08 \\
\hline & I 1.197 & 676.06 & 326.79 \\
\hline & 12.194 & .20 & .48 \\
\hline & 13.192 & .37 & .16 \\
\hline & 14.189 & .55 & 325.84 \\
\hline & I 5.186 & .75 & .53 \\
\hline & I6. 183 & .97 & .24 \\
\hline & I 7.I $8 I$ & 677.19 & $324.9^{6}$ \\
\hline & 18.178 & $.4 \mathrm{I}$ & .70 \\
\hline & I9.175 & .63 & .45 \\
\hline & 20.173 & .85 & .21 \\
\hline & 21.170 & 678.06 & 323.98 \\
\hline & 22.167 & .26 & .74 \\
\hline & 23.164 & .47 & .50 \\
\hline & 24.162 & .67 & .25 \\
\hline & 25.159 & .87 & .00 \\
\hline & 26.156 & 679.08 & 322.74 \\
\hline & 27.153 & .30 & .47 \\
\hline & $28.15 \mathrm{I}$ & .53 & . I 9 \\
\hline & 29.148 & .79 & 321.92 \\
\hline & 30.145 & 680.07 & .65 \\
\hline & 3 I.I 43 & .37 & .40 \\
\hline Áng. & I.I 40 & .67 & .18 \\
\hline & 2.137 & .98 & $3^{20.98}$ \\
\hline & 3.134 & $68 \mathrm{I} .27$ & .79 \\
\hline & 4.132 & .55 & $.6 \mathrm{I}$ \\
\hline & 5.129 & .82 & .42 \\
\hline & 6.126 & 682.08 & .22 \\
\hline & 7.123 & .33 & .02 \\
\hline & 8. I 2 I & .59 & $3 \times 9.79$ \\
\hline & 9.118 & .87 & $.5^{6}$ \\
\hline & 10.I I 5 & 683.16 & .32 \\
\hline & I I. I I 3 & .47 & .09 \\
\hline & I 2, I IO & .80 & 318.88 \\
\hline & 13.107 & 684.13 & .69 \\
\hline & I 4.104 & .46 & $.5 \mathrm{I}$ \\
\hline & 15.102 & .79 & .34 \\
\hline & I 6.099 & $685 . \mathrm{II}$ & .19 \\
\hline & 17.096 & .43 & .05 \\
\hline & I 8.093 & .74 & 317.90 \\
\hline & $\mathrm{I} 9.09 \mathrm{I}$ & 686.04 & .76 \\
\hline & 20.088 & .34 & .62 \\
\hline & 21.085 & .64 & .47 \\
\hline
\end{tabular}

\begin{tabular}{|c|c|c|}
\hline $\begin{array}{c}\text { Oatum in m. } \mathrm{Z} . \\
\text { Greenwich }\end{array}$ & $x$ & $y$ \\
\hline I92I & $1-$ & - \\
\hline ug. 22.082 & 686.94 & $317 \cdot 3 \mathrm{I}$ \\
\hline 23.080 & 687.25 & .14 \\
\hline 24.077 & .58 & 316.97 \\
\hline 25.074 & .92 & .80 \\
\hline 26.072 & 688.28 & .63 \\
\hline 27.06 .9 & .65 & .48 \\
\hline 28.066 & 689.04 & $\cdot 3^{6}$ \\
\hline 29.063 & .42 & .25 \\
\hline $30.06 \mathrm{I}$ & .80 & .16 \\
\hline $3^{1.058}$ & 690.17 & .09 \\
\hline ept. $\quad 1: 055$ & .53 & .02 \\
\hline 2.052 & .86 & 3 I 5.94 \\
\hline 3.050 & 69 I.I 9 & .85 \\
\hline 4.047 & .52 & .74 \\
\hline 5.044 & .86 & .62 \\
\hline 6.042 & 69.2 .22 & .50 \\
\hline 7.039 & .60 & .39 \\
\hline $8.03^{6}$ & .98 & .29 \\
\hline 9.033 & $693 \cdot 38$ & .20 \\
\hline 10.031 & .77 & . I 3 \\
\hline I 1.028 & $694 \cdot 16$ & .08 \\
\hline I 2.025 & .55 & .05 \\
\hline I 3.022 & .92 & .02 \\
\hline 14.020 & 695.29 & .00 \\
\hline I 5.017 & .65 & 314.98 \\
\hline 16.014 & 696.00 & .96 \\
\hline 1 7.012 & .35 & .93 \\
\hline I 8.009 & .69 & .90 \\
\hline I 9.006 & 697.04 & .86 \\
\hline 20.003 & .40 & $.8 \mathrm{I}$ \\
\hline $2 x .001$ & .77 & .77 \\
\hline 21.998 & 698.16 & .73 \\
\hline .22 .995 & .57 & .69 \\
\hline 23.992 & .98 & .68 \\
\hline 24.990 & $699.4^{\circ}$ & .69 \\
\hline 25.987 & $.8 \mathrm{I}$ & .72 \\
\hline 26.984 & 700.21 & .78 \\
\hline $27.98 \mathrm{I}$ & .59 & .83 \\
\hline 28.979 & .96 & .89 \\
\hline 29.976 & $7 \circ \mathrm{I} \cdot 3 \mathrm{I}$ & .93 \\
\hline 30.973 & .66 & .96 \\
\hline Okt. I.97 I & 702.01 & .98 \\
\hline 2.968 & $.3^{8}$ & .99 \\
\hline 3.965 & .76 & 3 I 5.00 \\
\hline 4.962 & $7 \circ 3 \cdot x 5$ & .02 \\
\hline 5.960 & $\quad .55$ & .06 \\
\hline
\end{tabular}

\begin{tabular}{|c|c|c|}
\hline $\begin{array}{c}\text { Datum in } \mathrm{m} . \mathrm{Z} . \\
\text { Greenwich }\end{array}$ & $x$ & $y$ \\
\hline 1921 & - & - \\
\hline Okt. $\quad 6.957$ & $7 \circ 3.95$ & 315.12 \\
\hline 7.954 & 704.35 & .19 \\
\hline $8.95 \mathrm{I}$ & .74 & .28 \\
\hline 9.949 & 705.12 & .39 \\
\hline 10.946 & .49 & .50 \\
\hline I I.943 & .84 & .61 \\
\hline I $2.94 \mathrm{I}$ & $706 . x 9$ & .72 \\
\hline I $3.93^{8}$ & .53 & .83 \\
\hline I 4.935 & .87 & .93 \\
\hline I 5.9 .32 & 707.20 & 316.03 \\
\hline
\end{tabular}

\begin{tabular}{ll|l}
16.930 & .54 & .12
\end{tabular}

$\begin{array}{ll}17.927 & .89\end{array}$

I 8.924708 .25

I $9.921 \quad .62$

20.919709 .01

$\begin{array}{lll}21.916 & .40 & .63\end{array}$

\begin{tabular}{ll|l}
22.9 & .78 & .79
\end{tabular}

\begin{tabular}{ll|l}
23.910 & 7 I0.1 $5 \quad .96$
\end{tabular}

\begin{tabular}{ll|l}
24.908 & $.5 \mathrm{I}$ & $3 \mathrm{I} 7.15$
\end{tabular}

\begin{tabular}{ll|l}
25.905 & .84 & .34
\end{tabular}

26.9027 I I.I 5

$27.900 \quad .46$

\begin{tabular}{l|l|}
28.897 & .77
\end{tabular}

29.8947 I 2.08

\begin{tabular}{ll|l}
$30.89 \mathrm{r}$ & .40 & $3 \mathrm{18.12}$
\end{tabular}

\begin{tabular}{r|r|r}
31.889 & .74 & .26 \\
1.886 & $7 \times 3.09$ & .42
\end{tabular}

\begin{tabular}{rr|r|r} 
Nov. & 1.886 & $7 \times 3.09$ & .42 \\
& 2.883 & .44 & .60
\end{tabular}

\begin{tabular}{ll|l}
2.883 & .44 & .60 \\
3.880 & .78 & .80
\end{tabular}

\begin{tabular}{l|l|l}
4.878 & 7 I 4.1 I & 3 I 9.0 I
\end{tabular}

\begin{tabular}{ll|l}
5.875 & .43 & .23
\end{tabular}

$\begin{array}{ll}6.872 \quad .74 & .47\end{array}$

$7.870715 .03 \quad .70$

\begin{tabular}{ll|l|l}
8.867 & $.3 \mathrm{I}$ & .94
\end{tabular}

\begin{tabular}{ll|l|l}
9.864 & .58 & 320.17
\end{tabular}

\begin{tabular}{l|r|r} 
I $0.86 \mathrm{I}$ & .84 & .40 \\
I 1.850 & 7 I 6.10 & .62
\end{tabular}

\begin{tabular}{r|r|r} 
I 1.859 & 7 I 6.10 & .62 \\
I 2.856 & .36 & .84
\end{tabular}

\begin{tabular}{ll|l} 
I 3.853 & .62 & 321.04
\end{tabular}

\begin{tabular}{r|r|r}
$\mathrm{I} 4.850$ & .89 & .25 \\
$\mathrm{I} 5.848$ & $7 \mathrm{I} 7.18$ & .46
\end{tabular}

\begin{tabular}{ll|l}
16.845 & .47 & .68
\end{tabular}

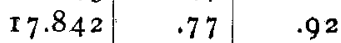

I $8.840 \quad 7$ I $8.06 \quad 322.19$

\begin{tabular}{l|l|l}
19.837 & .35 & .47
\end{tabular}

20.834

\begin{tabular}{c|c|c}
\hline $\begin{array}{c}\text { Datum in m. Z. } \\
\text { Greenwich }\end{array}$ & $x$ & $y$ \\
\hline 1921 & - & -
\end{tabular}

Nov. 2 I.83 I 7 I $8.85 \quad 323.07$ \begin{tabular}{ll|l|l}
22.829 & 719.07 & .37
\end{tabular}

\begin{tabular}{ll|l}
23.826 & .27 & .65
\end{tabular}

$24.823 \quad .47 \quad .92$

\begin{tabular}{l|l|l}
25.820 & .66 & 324.17
\end{tabular}

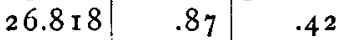

$27.8 \times 5 \times 720.09 \quad .66$

\begin{tabular}{ll|l}
$28.8 \times 2$ & .32 & .92
\end{tabular}

$29.809 \quad .55 \quad 325.19$

\begin{tabular}{ll|l}
30.807 & .77 & .48
\end{tabular}

\begin{tabular}{lr|r} 
Dez. & r.804 & .99 \\
\end{tabular}

2.801 72 I.19 326.10

$\begin{array}{lll}3.799 & .38 & .43\end{array}$

\begin{tabular}{ll|l}
4.796 & .55 & .76
\end{tabular}

\begin{tabular}{ll|l|l}
5.793 & .70 & 327.09
\end{tabular}

$\begin{array}{lll}6.790 & .84 & .41\end{array}$

\begin{tabular}{ll|l}
7.788 & .97 & .73
\end{tabular}

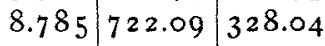

\begin{tabular}{ll|l}
9.782 & .21 & .34
\end{tabular}

\begin{tabular}{ll|l}
10.779 & .33 & .64
\end{tabular}

\begin{tabular}{ll|l|l}
$I I .777$ & .46 & .92
\end{tabular}

\begin{tabular}{l|l|l} 
I 2.774 & .59 & $329.2 \mathrm{I}$
\end{tabular}

\begin{tabular}{ll|l}
$\mathrm{I} 3.77 \mathrm{I}$ & .74 & .50
\end{tabular}

\begin{tabular}{l|l|l}
14.769 & .89 & .81
\end{tabular}

\begin{tabular}{ll|l|l}
15.766 & 723.04 & 330.14
\end{tabular}

\begin{tabular}{ll|l}
16.763 & .18 & .49
\end{tabular}

\begin{tabular}{ll|l}
$\mathrm{I} 7.760$ & .30 & .85
\end{tabular}

\begin{tabular}{ll|l}
18.758 & .39 & 331.21
\end{tabular}

\begin{tabular}{ll|l}
10.755 & .47 & .58
\end{tabular}

\begin{tabular}{ll|l}
20.752 & .52 & .93
\end{tabular}

$2 r .749 \quad .56 .332 .26$

\begin{tabular}{ll|l}
22.747 & .59 & .58
\end{tabular}

\begin{tabular}{ll|l}
23.744 & .63 & .88
\end{tabular}

\begin{tabular}{l|l|l}
24.741 & .68 & 333.17
\end{tabular}

\begin{tabular}{ll|l}
25.739 & .74 & .47
\end{tabular}

\begin{tabular}{ll|l}
26.736 & .80 & .78
\end{tabular}

\begin{tabular}{ll|l}
27.733 & .86 & $334 . \mathrm{I}$
\end{tabular}

\begin{tabular}{ll|l|l}
28.730 & .92 & .45
\end{tabular}

\begin{tabular}{ll|l}
29.728 & .96 & .80
\end{tabular}

\begin{tabular}{ll|l|l}
30.725 & .98 & 335.16
\end{tabular}

\begin{tabular}{lr|r}
31.722 & .98 & .52
\end{tabular}

\begin{tabular}{ll|l}
32.719 & .96 & .88
\end{tabular}

\begin{tabular}{l|l|l}
33.717 & .94 & $33^{6.23}$
\end{tabular}

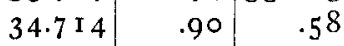

\begin{tabular}{ll|l}
35.7 I I & .85 & .91
\end{tabular}

\section{Courvoisier.}

Berlin-Babelsberg, I 920 Nov, I 7 .

\title{
Über 3 Algolsterne und ihre besonderen Perioden.
}

Der schwächste der 4 Hauptsterne des Trapez im großen Orionnebel, BM Orionis, von dem Dr. Goos i 9 I 8 die Mitteilung machte, daß er ihn zweimal, I 17 Febr. I 4 und 1918 Febr. I 7 , schwächer als sonst gesehen hätte, hat sich nach meiner Überwachung als ein Algolstern erwiesen, der alle $6 \%$ Tage eine Verfinsterung erfährt. Der Umstand, daß die Periode nahe ein ganzes Vielfaches von einem halben Tag ist, hat zur
Folge, daß sich die Zeiten kleinsten Lichtes lange Zeit hin- . durch nicht bestimmen lassen, indem sie, wie in diesem Herbst I920, alle I 3 Tage gerade mit der Aufgangszeit, im Frühjahr I 92 I mit. der Untergangszeit zusammenfallen. Im Frühjahr I 920 konnte ich noch ankündigen, daß am I 1 . und 24. April eine Verfinsterung eintritt. Sie müßte beim Untergang in der Abenddämmerung eingetreten sein. Ich habe 
nun am 9. Okt. I920 bei vom Ostwind veranlaßten außerordentlich schlechten Bildern eine sehr unsichere Bestimmung des kleinsten Lichtes für $\mathrm{I} 4^{\text {h }}$ erhalten, die möglicherweise $\mathrm{um}$ Stunden falsch sein kann, weil die zunehmende Verschlechterung derBilder die Beobachtung abzubrechen zwang, ehe ein Aufstieg angedeutet war. Die vorjährigenBeobachtungendes Sterns hatten auch unter schlechten Luftverhältnis-

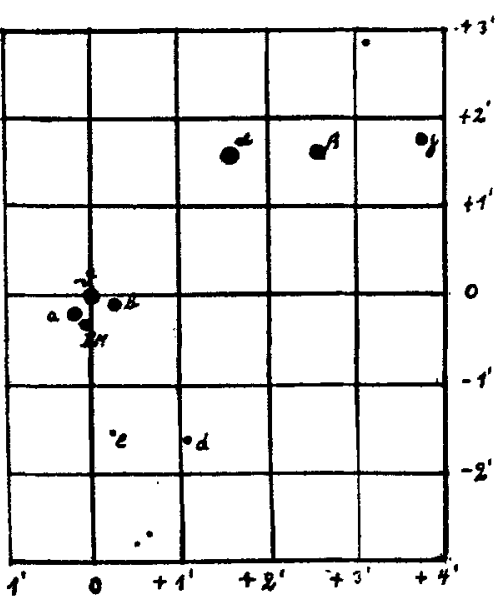
sen gelitten, die bei diesem Stern wegen der Nähe der hellen Sterne und des nebligen Untergrundes besonders störend werden. Von den beiden von Herrn Dr. Goos mitgeteilten Tagen, die keine Stundenangabe enthalten, scheint der erste nicht richtig $\mathrm{zu}$ sein und um einen Tag früher verlegt werden zu müssen. Wenn ich nun auch weder über die Dauer der Verfinsterung einen genauen Wert anzugeben vermag, noch überhaupt ein kleinstes Licht bisher ableiten konnte, so möchte ich doch auf die nächsten Gelegenheiten zur Beobachtung wenigstens eines der beiden Lichtäste, also entweder des Aufstiegs oder des Abstiegs aufmerksam machen, um bei der Untersuchung dieses merkwürdigen Algolsterns durch gemeinsame Verfolgung an verschiedenen Orten von der Witterung unabhängiger zu werden. Daß die Veränderlichkeit nicht schon früher bemerkt worden ist, darf wohl der ungünstigen Periode zugeschrieben werden, die längere Zeit hindurch die Verfinsterungen, wie jetzt, in die Zeit des Aufgangs oder Untergangs fallen läßt und immer nur alle I 3 Tage Gelegenheit zu ibrer Wabrnehmung darbietet. Bisher habe ich noch keine größere Lichtschwächung als $0^{\mathrm{m}} \cdot 75$ bemerken können. Die Angabe, daß sie bis zur i r. Größe geht, ist durch den Gebrauch kleiner Fernröhre wohl veranlaßt, die wegen der nahen hellen Sterne den geschwächten Stern nicht mehr deutlich zeigen. Wenn nach den bis jetzt aufstellbaren Elementen $m=2422199^{\mathrm{d}} 576+6{ }^{\mathrm{d}} \cdot 4754 \cdot E$ (I919 Aug. 28) die jetzt beobachtbaren Verfinsterungen als ungerade Epochen erscheinen, so werden von Januar I92 I

Bamberg, I 920 Nov. II. ab die geraden Epochen wahrnehmbar. Die Epochen sind: .

\begin{tabular}{crrrr}
$E$ & I920-2I & \multicolumn{3}{c}{ M. Z. Gr. } \\
69 & Nov. I 7 & $9^{\mathrm{h}}$ & $5^{\mathrm{m}}$ \\
70 & & 23 & 20 & 30 \\
$7 \mathrm{I}$ & & 30 & 7 & 54 \\
72 & Dez. & 6 & 19 & I 9 \\
73 & & I 3 & 6 & 44 \\
74 & & I 9 & I 8 & 8 \\
75 & 26 & 5 & 33 \\
76 & Jan. & I & I6 & 57 \\
77 & 8 & 4 & 22 \\
78 & I 4 & I 5 & 46 \\
79 & 21 & 3 & 11 \\
80 & 27 & I 4 & 36
\end{tabular}

\begin{tabular}{|c|c|c|c|c|}
\hline$E$ & \multicolumn{2}{|l|}{1921} & \multicolumn{2}{|c|}{ M. Z. Gr. } \\
\hline 81 & Febr. & 3 & & $0^{\text {tr }}$ \\
\hline 82 & & 9 & I 3 & 25 \\
\hline 83 & & I 6 & 0 & 49 \\
\hline 84 & & 22 & I 2 & I 4 \\
\hline 85 & & 28 & 23 & 38 \\
\hline 86 & März & 7 & II & 3 \\
\hline 87 & & I 3 & 22 & 28 \\
\hline 88 & & 20 & 9 & 52 \\
\hline 89 & & 26 & 2 I & I 7 \\
\hline 90 & April & 2 & 8 & $4 I$ \\
\hline 9 I & & 8 & 20 & 0 \\
\hline 92 & & I 5 & 7 & 30 \\
\hline
\end{tabular}

Der zweite Algolstern ist 77.I9I9 WX Andromedae I $8550^{\mathrm{h}} 37^{\mathrm{m}} 53^{\mathrm{s}}+27^{\circ} 57^{\prime} 3$, der von Wolf als ein rasch wechselnder Verdunkelungsveränderlicher angezeigt ist, Die Algolart ist hier in Bamberg bestätigt worden. Die Elemente sind $m=2422547^{\text {d. }} 53$ 1 $25+3^{\text {d }}$.00104 I. E (1920 Aug. Io). Es fallen die Verfinsterungen gegenwärtig auf die späten Nachtstunden und lassen keine Möglichkeit, den Aufstieg des Lichtes zu beobachten. Auch hier veranlaßt der Umstand, daß die Periode ein ganzes Vielfaches von einem halben Tag ist, durch lange Zeit unbeobachtbare Zeiten kleinsten Lichtes. Die $A b$ - und Zunahme nimmt etwa io Stunden in Anspruch, ähnlich wie bei Algol, dessen Periode auch nahe 3 Tage beträgt. Die nächsten Verfinsterungen finden in den späten Nachtstunden von Nov. I 4, I 7, 20, 23, 26, 29, Dez. 2, 5,8, I I, I $4, \mathrm{r}_{7}, 20,23,26$ und 29 statt.

Der dritte Algolstern ist der hier entdeckte VZ Aurigae I $8556^{\mathrm{h}} 3^{\mathrm{m}} 37^{\mathrm{s}}+4^{\circ} 6^{\mathrm{r}} \mathrm{\prime} \cdot 2$, der auf der Karte 4 der in AN I74.364 von Dr. Silbernagel mitgeteilten 4 Veränderlichen in Auriga mit dem Buchstaben $a\left(\mathrm{BD}+46^{\circ} \mathrm{I} \perp \mathrm{I}, 9^{\mathrm{m}} \cdot 5\right)$ bezeichnet ist. Seine Periode ist nahezu 24 Stunden. Es war mir bisher noch nicht möglich, die volle Lichtkurve durchzubeobachten. Jetzt sind die Zeiten kleinsten Lichtes in frühere Nachtstunden gerückt und können daher in jeder hellen Nacht aus Abstieg und Aufstieg festgelegt werden. Es sollte diese Zeit ausgenützt werden, - weil später Jahre hindurch ihre Beobachtung unmöglich ist, da die nahe Gleichheit der Periode mit einem ganzen Vielfachen eines halben Tags sie nur langsam, wenn sie in Tagesstunden gelangt sind, aus ihnen wieder herausrücken läßt.

\section{E. Hartwig.}

\section{Aufforderung betreffend die Erneuerung der Bestellung auf die Beobachtungs-Zirkulare.}

Die bisherigen Bezieher der Beobachtungs-Zirkulare, soweit sie nicht fortlaufende, ununterbrochene Zusendung ausdrücklich verlangt haben, werden ersucht ihre Bestellung auf den Jahrgang I 92 I umgehend einzusenden, damit eine Unterbrechung der Zusendung vermieden werde. Für die nicht als ständige Bezieher eingeschriebenen Empfänger liegt ein Bestellformular bei.

In den Beobachtungs-Zirkularen werden die zur Ausführung der Beobachtung stattfindender oder nahe bevorstehender Himmelserscheinungen erforderlichen Hilfsmittel veröffentlicht und kurze, vorläufige zu weiterer Verfolgung oder Mitarbeit anregende Berichte über ausgeführte Arbeiten gegeben. Der Inhalt der Zirkulare kann in den Astronomischen Nachrichten selbst nur dann wiedergegeben werden, wenn es sich um Mitteilungen über in abgeschlossener Form vorliegende Resultate handelt.

Der Preis der Zirkulare, deren Ausgabe in ihrem $Z$ wecke entsprechenden Abständen, in der Regel einmal in der Woche, erfolgt, ist bei gleichzeitigem Bezug der Astronomischen Nachrichten durch die Expedition 15 M. für den Jahrgang. Für Nichtbezieher der Zeitschrift muß der Preis auf zo $M$. für den Jahrgang erhöht werden. Der Herausgeber.

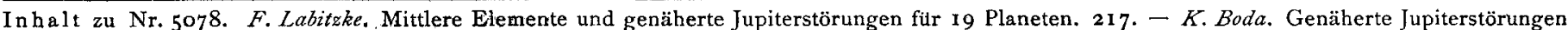
für 108 Planeten der Hestiagruppe. 219. - L. Courvoisier. Ephemeride der Polarissma (BD $\left.+89^{\circ} 37\right)$ fiur 1921. 227. E. Hartwig. Über 3 Algolsterne und ihre besonderen Perioden. 229, - Aufforderung betreffend die Erneuerung der Bestellung auf die Beobachtungs-Zirkulare. $23 \mathbf{I}$.

Geschlossen t920 Dez. 22. Herausgeber: H. Kobold. Druck von C.Schaidt. Expedition: Kiel, Moltkestr. 80. Postscheck-Konto Nr. 6z38 Hamburg II. 